\title{
Introducing the EBM Verdict: research evidence relevant to clinical practice
}

\section{Carl Heneghan, ${ }^{1}$ Jon Brassey, ${ }^{2}$ Jeffrey Aronson, ${ }^{1}$ Jack William O'Sullivan ${ }^{\oplus 1,3}$}

10.1136/bmjebm-2019-111172

${ }^{1}$ Nuffield Department of Primary Care Health Sciences, Centre for Evidence Based Medicine, University of Oxford, Oxford, UK

${ }^{2}$ Trip Database Ltd, Newport, UK

${ }^{3}$ Division of Cardiology, Department of Medicine, Stanford University, Palo Alto, California, USA

Correspondence to: Professor Carl Heneghan, Centre for Evidence Based Medicine, University of Oxford Oxford UK ; carlheneghan@ bmj.com

\section{Check for updates}

( $)$ Author(s) (or their employer(s)) 2019. No commercial re-use. See rights and permissions. Published by BMJ.

To cite: Heneghan C, Brassey J, Aronson J, et al. BMJ Evidence-Based Medicine 2019;24:85-86.
The BMJ Evidence-Based Medicine Journal was launched in 1995, with the purpose of alerting clinicians to important advances in medicine, by selecting original articles and reviews whose results were most likely to be both accurate and useful. $^{1}$

When the Journal was launched, about 10500 randomised trials were indexed on PubMed. Identifying the trials that affect practice has become harder: 20 years later, over 30000 trials are published annually. If we focused purely on systematic reviews, we would face similar problems: over 19000 systematic reviews were indexed on PubMed in 2017. Identifying the evidence that matters, keeping up to date and applying evidence in practice is a significant challenge for busy clinicians.

As a result, we as a journal have set out to identify, and focus on, the research evidence that provides definitive conclusions and research that confirms, refutes or improves current practice.

We have focused on two questions: (1) does this research apply to the patients we see in practice? and (2) what difference could this evidence make to my patient? In doing so, we can remove a substantial amount of research that does not matter. Much of it does not include patients typically seen in clinical practice, limiting the opportunity to influence practice. Determining the difference a treatment makes at the outset can, however, allow for more rapid assessment of the evidence, discarding what does not make a difference to patient care and focussing on the research that does. A large proportion of papers detailing research evidencealthough we're not sure how much-conclude that more research is needed, or that an intervention or test should not be implemented in practice.

Our new series of EBM Verdict will, therefore, feature only the evidence that we consider comes to a definitive conclusion: the evidence that can be implemented immediately in clinical practice.

To create interesting, clearly written articles that cover the thought-provoking, controversial or essential points relevant to practice, our EBM Verdict articles will include information on the purpose of the study, why the study was needed and what clinical uncertainty led to the study question. We shall criticise a study when necessary, and we shall discuss its implications for practice and healthcare. We will use absolute effects to explain the results-unaccompanied relative measures will be banned. We will be specific in our recommendations and consider the uncertainty in the evidence and its relevance to real-world patients and decisions.

This week Igho Onakpoya and Jeff Aronson publish the first in the EBM Verdict series of articles (10.1136/bmjebm-2018-111128). Lorcaserin in obesity shows minimal benefits and ill-defined harms. The EBM Verdict concludes that the minimal weight reductions observed and the selective reporting of harms leave major uncertainties about Lorcaserin's benefit-harm profile, and because the harms profile is as yet incompletely understood, it is unlikely to be a cost-effective intervention.

Our plan is to publish a practice-changing EBM Verdict article weekly. We will aim to get the articles up quicker when there is a pressing need. We will also supplement the clinical pieces with EBM methods Verdict. Led by Rafael Perera, our statistics editor, we plan to scan for the methods that make a difference to the production, interpretation, and publication of evidence for better healthcare. How do you find practice-changing research evidence? In our BMJ EBM Spotlight Blog, Jon Brassey has set out the relevant search processes. $^{2}$

Our EBM Verdict pieces will reach definitive conclusions and answer straight-forward questions-should an intervention or test be started (or stopped) in practice? We will not conclude with remarks such as more research is needed' or 'these results might..... And is this research robust-are the conclusions based on high-quality research? We will harness existing systems to support article selection. For instance, there are already systems, such as EvidenceAlerts ${ }^{3}$ and NIHR Signals, ${ }^{4}$ which filter the mass of journal articles. We then scan the five internal medical journals (BMJ, NEJM, Lancet, JAMA and Annals of Internal Medicine). Again, we use the criteria of definitive conclusions as inclusion criteria for the long list.

The EBM Verdict process is iterative, so we would appreciate your thoughts as we go along this journey. As we become clearer in how and what to select, we will grow the number of contributors. We look forward to your feedback.

Acknowledgements Our Editorial Board that produce the EBM verdict comprises of Professor CH, Dr Igho Onakpoya, Dr JA, JB, Dr Denise Campbell-Scherer, Professor Khalid Khan, Associate Professor Kamal Mahtani, Dr JO'S, Dr Annette Plüddemann and Professor Rafael Perera. 


\section{Editorial}

Funding The authors have not declared a specific grant for this research from any funding agency in the public, commercial or not-for-profit sectors.

Competing interests $\mathrm{CH}$ is the Professor of Evidence-Based Medicine and Director of the Centre for Evidence-Based Medicine (CEBM), University of Oxford. He has received expenses and fees for his media work and holds grant funding from the NIHR, the NIHR School of Primary Care Research and The NIHR Oxford BRC. CEBM, part of the at the Nuffield Department of Primary Care Health Sciences (NDPHCS), jointly runs the Evidence Live Conference with the BMJ and the Overdiagnosis Conference with some international partners which are based on a nonprofit model. JB runs the search engine the Trip Database (www. tripdatabase.com) and owns 50\% of the shares in the company and is an Associate Editor at BMJ EBM. KRM is an Associate Professor at CEBM, University of Oxford, receives funding from the NIHR SPCR Evidence Synthesis Working Group and the NIHR Health Technology Assessment programme and is an Associate Editor at BMJ EBM. JOS is a Postdoctoral Fellow at Stanford
University. His Fellowship is funded by the National Institutes of Health. He is an Associate Editor of BMJ EBM. JA is a is a clinical pharmacologist at CEBM, University of Oxford. He is also president emeritus of the British Pharmacological Society and an Associate Editor at BMJ EBM.

Patient consent for publication Not required.

Provenance and peer review Not commissioned; internally peer reviewed.

\section{References}

1. Website. https://ebm.bmj.com/content/1/1/5 (Accessed 17 Jan 2019).

2. Brassey J. How do you find practice-changing research evidence? | BMJ EBM Spotlight. BMJ EBM Spotlight 2018 https://blogs.bmj.com/ bmjebmspotlight/2018/12/03/how-do-you-find-practice-changing-researchevidence/ (Accessed 17 Jan 2019).

3. EvidenceAlerts. https://plus.mcmaster.ca/evidencealerts/ (Accessed 17 Jan 2019).

4. Centre ND. Search NIHR Signals | Summaries of important health research evidence. https://discover.dc.nihr.ac.uk/portal/search/signals (Accessed 17 Jan 2019). 